\title{
Alum Catalyzed Simple, Efficient, and Green Synthesis of 2-[3-Amino-5-methyl-5-(pyridin-3-yl)-1,5-dihydro-4H-1,2,4- triazol-4-yl]propanoic Acid Derivatives in Aqueous Media
}

\author{
Harshita Sachdeva, Diksha Dwivedi, and Rekha Saroj \\ Department of Chemistry, Faculty of Engineering and Technology, Mody Institute of Technology and Science, \\ Lakshmangarh, Sikar, Rajasthan 332311, India
}

Correspondence should be addressed to Harshita Sachdeva; drhmsachdevaster@gmail.com

Received 4 August 2013; Accepted 8 September 2013

Academic Editors: G. C. Vougioukalakis, A. D. Wastowski, and M. Wujec

Copyright (c) 2013 Harshita Sachdeva et al. This is an open access article distributed under the Creative Commons Attribution License, which permits unrestricted use, distribution, and reproduction in any medium, provided the original work is properly cited.

Alum $\left(\mathrm{KAl}\left(\mathrm{SO}_{4}\right)_{2} \cdot 12 \mathrm{H}_{2} \mathrm{O}\right)$ is an inexpensive, efficient, and nontoxic catalyst used for the synthesis of 2-[3-amino-5-methyl-5(pyridin-3-yl)-1,5-dihydro-4H-1,2,4-triazol-4-yl]propanoic acid derivatives in aqueous media by the reaction of 3-acetyl pyridine (1), amino acids $(2) /(6)$, and thiosemicarbazide (4) at $80^{\circ} \mathrm{C}$. This methodology offers significant improvements for the synthesis of products with regards to the yield of products, simplicity in operation, and green aspects by avoiding toxic catalysts which uphold the motto of green chemistry. Synthesized compounds have been characterized by FT-IR, ${ }^{13} \mathrm{C}$ NMR, and ${ }^{1} \mathrm{HNMR}$ spectroscopy.

\section{Introduction}

In the last two decades, it has become increasingly clear that the chemical industries are facing serious environmental problems. Many of the classical synthetic methodologies have a broad scope but generate copious amounts of waste, and the chemical industry has been subjected to increasing pressure to minimize or, preferably, eliminate this waste. Many organic solvents are hazardous and can be deleterious to human health. They are volatile and cause an environmental threat by polluting the atmosphere. Green chemistry approaches are significant due to reduction in byproducts, waste produced, and energy cost. In recent years, organic research is mainly focused on the development of green methods which involve the use of alternative reaction media to replace volatile and hazardous solvents commonly used in organic synthesis. In this regard, the use of water as a reaction solvent has also attracted great attention and has become an active area of research in green chemistry.

Nowadays, many organic transformations have been carried out in water [1-3]. It is a unique solvent due to being readily available, inexpensive, nontoxic, safer, and environmentally benign. The aqueous mediated conditions lead to enhanced reaction rates, higher yields of pure products, and easier workup and sometimes to selective conversions with several advantages of the ecofriendly approach in the framework of green chemistry. Consequently, this protocol should be welcomed in these environmentally conscious days.

Catalysis cannot only help to green chemical processes (e.g., by replacing reagents or by enabling more efficient processes), but the demonstration of their value to reduce the environmental impact of processes and reduce the costs of the processes will catalyze the greening of chemistry. In recent years, alum $\left(\mathrm{KAl}\left(\mathrm{SO}_{4}\right)_{2} \cdot 12 \mathrm{H}_{2} \mathrm{O}\right)$ is extensively used as a catalystin organic synthesis because it is a nontoxic, inexpensive, ecofriendly, and easy handling catalyst. Other advantages include mild acidity, involatility, incorrositivity, insolubility in common organic solvents, and so forth. Alum has previously been reported to be effective in the synthesis of 1,4-dihydropyridines [4], cis-isoquinolic acids [5], monoand disubstituted 2,3-dihydroquinazolin-4(1H)-ones [6], dihydropyrimidine via Biginelli reaction [7], coumarins [8], 5-arylidene-2,4-thiazolidinedione [9], dibenzoxanthenes [10], 1,5-benzodiazepines [11], and trisubstituted imidazoles [12]. We investigated alum as a catalyst for 


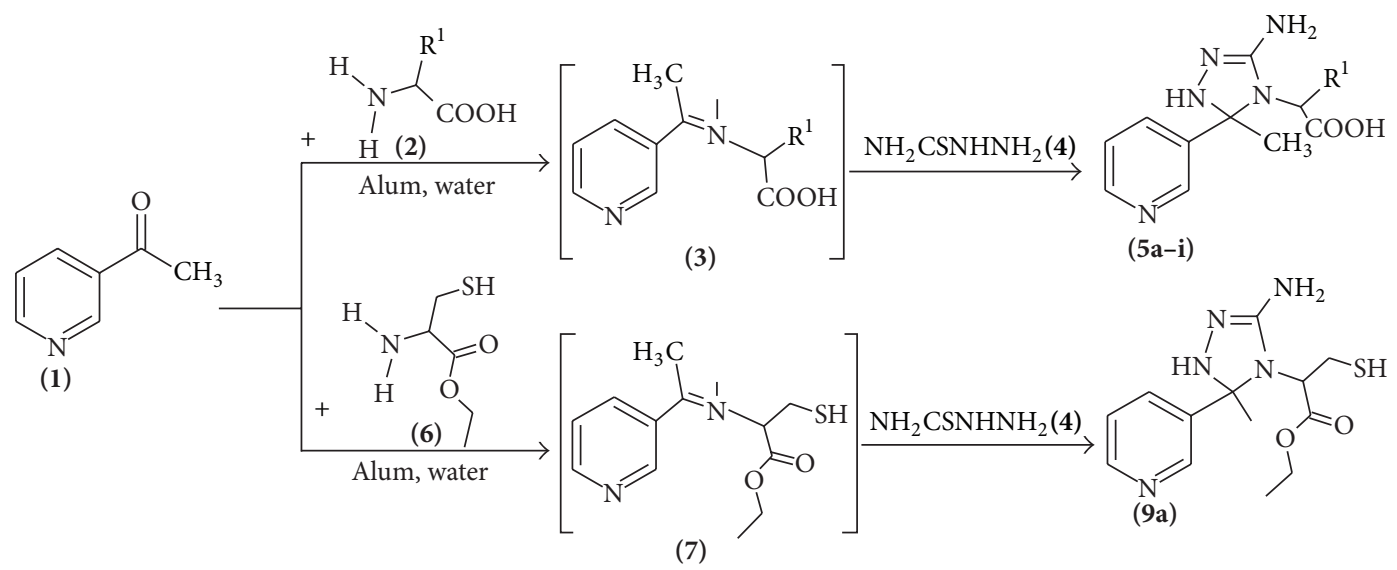

SCHEME 1

the synthesis of 2-[3-amino-5-methyl-5-(pyridin-3-yl)-1,5dihydro-4H-1,2,4-triazol-4-yl]propanoic acid derivatives $(5 \mathbf{a}-\mathbf{i})$ by the reaction of 3 -acetyl pyridine and amino-acids with thiosemicarbazide.

The search for new agent is one of the most challenging tasks to the medicinal chemist. The synthesis of high nitrogen containing heterocyclic system has been attracting increasing interest because of its utility in various applications, such as propellants, explosives, pyrotechnics, and especially chemotherapy. There is real perceived need for the discovery of new compounds endowed with antimicrobial activity, possibly acting through mechanisms of action, which are distinct from those of well-known classes of antibacterial agents to which many clinically relevant pathogens are now resistant. 1,2,4-Triazoles represent an overwhelming and rapid developing field in modern heterocyclic chemistry.

From the literature, it is predictable that 1,2,4-triazoles represent important pharmacophores and play a vital role as medicinal agents. A degree of respectability has been bestowed for 1,2,4-triazole derivatives due to their wide range of biological activities such as antimicrobial [13-15], analgesic, anti-inflammatory [16, 17], anticancer [18] and antioxidant properties [19]. Ribavirin (antiviral) [20] rizatriptan (antimigraine) [21], posaconazole, fluconazole, and Itraconazole $[22,23]$ are efficient antifungal drugs currently used in the treatment of fungal infection. Several articles devoted to the synthesis and biological activity of arylidenaminotriazoles have been published [24-29] due to their potential biological activities. In view of the above observations, an attempt has been made to synthesise 2-[3-amino-5-methyl-5-(pyridin-3-yl)-1,5-dihydro-4H-1,2,4-triazol4-yl]propanoic acid derivatives $(5 \mathbf{a}-\mathbf{i}) /(9 a)$ (Scheme 1) by treating 3 -acetyl pyridine (1) and amino acids $(2) /(6)$ with thiosemicarbazide (4) under the framework of green chemistry using alum as a green catalyst in water.

\section{Results and Discussion}

In continuation to our work on the development of green methodologies [30-32] for heterocyclic synthesis, herein we would like to report a simple, efficient, and rapid method for the synthesis of triazole derivatives (5a-i)/(9a) (Table 1). The condensation of 3 -acetyl pyridine with various amino acids/cysteine amino acid yielded 2-\{[1-(pyridin-3yl)ethylidene] amino\}propanoic acid (3)/ethyl 2-\{[1-(pyridin3-yl)ethylidene]amino\}-3-sulfanylpropanoate (7) which "insitu" were cyclocondensed with thiosemicarbazide (4) in the presence of alum in aqueous medium to give exclusively 5a-i/9a, respectively, in $86-90 \%$ (Scheme 1) (Table 1). We have recently reported [33] the environ-economic synthesis of some new 1,2,4-triazole derivatives by the reaction of 4-chloro-2-nitro aniline and aromatic aldehydes with thiosemicarbazide in high yields and shorter reaction time in the presence of lemon juice as a green catalyst.

The reaction was extensively studied under different reaction conditions to find out the best method giving the product in higher yield and short reaction time with operational simplicity. As shown in Tables 2, 3, and 4, the reaction of 3-acetyl pyridine (1) and alanine amino acid (2) with thiosemicarbazide (4) was chosen as the model substrate to optimize reaction conditions including effect of temperature, type of catalyst, and concentration of catalyst. Table 2 shows the effect of temperature on the formation of compound $\mathbf{5 a}$ in the presence of alum as a green catalyst. It is observed that the rate of reaction increases on increasing the temperature due to increase in acidity and the best yield is obtained at $80^{\circ} \mathrm{C}$ temperature in a short reaction time of $6 \mathrm{hrs}$. The acidity of the alum depends highly on the quantity of trapped water molecules in the interlayers. Alums liquefy on heating and if the heating is continued, the water of crystallization is driven off, the salt froths and swells, causing decrease in Bronsted acidity but increase in Lewis acidity. Hence other compounds were also synthesized under similar reaction conditions (at $\left.80^{\circ} \mathrm{C}\right)$.

In Table 3, our results are compared with results obtained by other catalysts for the synthesis of compound 5a. The data presented in this Table 3 shows the promising features of this method in terms of the yield of the product compared to other catalysts. Other catalysts, namely, silica, alumina, and phosphorus pentoxide were also screened at $80^{\circ} \mathrm{C}$ (Table 3 ) (entries 1-3), and the results show that the alum provided the highest yield (entry 4) (Table 3 ). Notably, a very slow 
TABLE 1: Physical characterization data of compounds (5a-i) and (9a).

\begin{tabular}{|c|c|c|c|c|c|}
\hline Entry & $\mathrm{R}^{1}$ & Products & Time (hrs) & Yield (\%) & $\mathrm{MP}\left({ }^{\circ} \mathrm{C}\right)$ \\
\hline $5 a$ & $-\mathrm{CH}_{3}$ & & 6 & 90 & 200 \\
\hline $5 \mathbf{b}$ & $-\mathrm{CH}_{2} \mathrm{COOH}$ & & 7 & 87 & 205 \\
\hline $5 c$ & $\begin{array}{c}-\mathrm{CH}_{2}\left(\mathrm{CH}_{3}\right) \\
\mathrm{CH}_{2} \mathrm{CH}_{3}\end{array}$ & & 7 & 87 & 194 \\
\hline $5 d$ & & & 8 & 88 & 174 \\
\hline $5 e$ & $-\mathrm{CH}_{2} \mathrm{CH}_{2} \mathrm{SCH}_{3}$ & & 6 & 90 & 185 \\
\hline $5 f$ & $-\mathrm{CH}_{2} \mathrm{C}_{6} \mathrm{H}_{5}$ & & 7 & 88 & 182 \\
\hline $5 \mathrm{~g}$ & $-\mathrm{CH}_{2} \mathrm{OH}$ & & 6 & 89 & 198 \\
\hline $5 \mathrm{~h}$ & & & 6 & 86 & 197 \\
\hline $5 i$ & $\mathrm{CH}_{2}\left(\mathrm{CH}_{3}\right)_{2}$ & & 7 & 87 & 183 \\
\hline
\end{tabular}


TABle 1: Continued.

\begin{tabular}{lllll}
\hline Entry & $\mathrm{R}^{1}$ & Time (hrs) & Yield (\%) \\
$9 \mathrm{a}$ & - & $\mathrm{MP}\left({ }^{\circ} \mathrm{C}\right)$ \\
\hline
\end{tabular}

Reaction Conditions: All the compounds were synthesized by stirring 3-acetyl pyridine $\mathbf{1}$ ( $2 \mathrm{mmol})$, amino acid $\mathbf{2}$ ( 2 mmol), thiosemicarbazide ( $2 \mathrm{mmol})$, and alum $(25 \mathrm{~mol} \%)$ in water $(25 \mathrm{~mL})$ at $80^{\circ} \mathrm{C}$ for $6-7 \mathrm{hrs}$.

TABLE 2: Effect of temperature on the formation of $\mathbf{5 a}$ in the presence of alum as catalyst.

\begin{tabular}{lccc}
\hline Entry & Temperature $\left({ }^{\circ} \mathrm{C}\right)$ & Time $(\mathrm{hrs})$ & Yield (\%) \\
\hline $\mathbf{1}$ & Room temp. & 25 & Nil \\
$\mathbf{2}$ & 50 & 18 & 40 \\
$\mathbf{3}$ & 60 & 15 & 56 \\
$\mathbf{4}$ & 70 & 10 & 66 \\
$\mathbf{5}$ & 80 & 6 & 90 \\
$\mathbf{6}$ & 100 & 6 & 90 \\
\hline
\end{tabular}

TABLE 3: Effect of various catalysts on the formation of $\mathbf{5 a}$ at $80^{\circ} \mathrm{C}$.

\begin{tabular}{lccc}
\hline Entry & Catalyst & Time (hrs) & Yield (\%) \\
\hline $\mathbf{1}$ & $\mathrm{SiO}_{2}$ & 15 & 40 \\
$\mathbf{2}$ & $\mathrm{Al}_{2} \mathrm{O}_{3}$ & 10 & 63 \\
$\mathbf{3}$ & $\mathrm{P}_{2} \mathrm{O}_{5}$ & 8 & 75 \\
$\mathbf{4}$ & $\mathrm{Alum}$ & 6 & 90 \\
\hline
\end{tabular}

TABLE 4: Effect of alum catalyst loading for synthesis of $\mathbf{5 a}$ at $80^{\circ} \mathrm{C}$.

\begin{tabular}{lcc}
\hline Entry & Catalyst $(\mathrm{mol} \%)$ & Yield $(\%)$ \\
\hline $\mathbf{1}$ & 10 & traces \\
$\mathbf{2}$ & 15 & 50 \\
$\mathbf{3}$ & 20 & 86 \\
$\mathbf{4}$ & 25 & 90 \\
$\mathbf{5}$ & 30 & 90 \\
\hline
\end{tabular}

reaction was observed when the catalytic amount of alum was decreased from 15 to $10 \mathrm{~mol} \%$ (entry 2 versus entry $\mathbf{1}$ ) (Table 4). When the catalytic amount of alum is increased from 15 to $20 \mathrm{~mol} \%$, a large increase in yield is observed (entry 2 versus entry 3 ). With 25 to $30 \mathrm{~mol} \%$ of alum, there is no change in reaction rate as well as yield of the product (entry 4 versus entry 5). Further, there is an increase in $4 \%$ yield when mol\% of alum is increased from 20 to $25 \%$ (entry 3 versus entry 4).

Herein, we have developed an efficient methodology for the synthesis of triazole derivatives $(5 \mathbf{a}-\mathbf{i})$ using alum as a green catalyst in aqueous medium at $80^{\circ} \mathrm{C}$. The methodology developed is simple giving product in excellent yields. To investigate the generality of the reaction, various substituted amino acids were studied, all of which undergo smooth reactions without the formation of any byproduct (Table 1) as observed on TLC.

\section{Experimental}

Chemicals were purchased from Sigma-Aldrich and Merck and used without further purification. Melting points were determined on an Instrument India Melting Point Apparatus. The spectral analyses of synthesized compounds have been carried out at SAIF, Punjab University, Chandigarh. Monitoring the reactions and checking the purity of the final products were carried out by thin layer chromatography (TLC) on silica gel $G$ plates using benzene : ethyl acetate $(7: 3 \mathrm{v} / \mathrm{v})$ as eluent. IR spectra were recorded in $\mathrm{KBr}$ on a Perkin Elmer Infrared L1600300 Spectrum Two Li Ta spectrophotometer. ${ }^{1} \mathrm{H}$ and ${ }^{13} \mathrm{C}$ NMR spectra were recorded on Bruker Avance II 400 NMR Spectrometer using DMSO as solvent and tetramethylsilane (TMS) as internal reference standard. The obtained products were identified from their spectral $\left({ }^{1} \mathrm{H}\right.$ $\mathrm{NMR},{ }^{13} \mathrm{C}$ NMR and IR) analyses.

3.1. General Procedure for the Synthesis of 2-[3-Amino-5methyl-5-(pyridin-3-yl)-1,5-dihydro-4H-1,2,4-triazol-4-yl]propanoic Acid Derivatives $(\mathbf{5} \boldsymbol{a}-\mathbf{i}) / \mathbf{9 a}$. The compounds were synthesized by the reaction of 3-acetyl pyridine and various amino acids with thiosemicarbazide in aqueous medium using alum as an ecofriendly catalyst. In a round bottom flask was placed a mixture of 3-acetyl pyridine 1 ( $2 \mathrm{mmol})$, amino acid $2(2 \mathrm{mmol})$, and alum $(25 \mathrm{~mol} \%)$ in water $(25 \mathrm{~mL})$. The suspension was stirred at $80^{\circ} \mathrm{C}$ for a certain period of time required to complete the reaction (as monitored by TLC). As the reactants disappeared, $2 \mathrm{mmol}$ of thiosemicarbazide was added and again stirred at $80^{\circ} \mathrm{C}$ for appropriate time. After the completion of reaction, the obtained product was filtered, washed with cold water, and recrystallized from ethanol.

\subsection{Characterization of the Compounds is Carried out on the Basis of Spectral Data}

3.2.1. 5a. 2-[3-Amino-5-methyl-5-(pyridin-3-yl)-1,5-dihydro4H-1,2,4-triazol-4-yl]propanoic Acid. IR $\left(\mathrm{KBr}, \mathrm{cm}^{-1}\right) 3348$, 3258, 2854, 2560, 1670, 1546, 1416, 1343, 1187, 1064, 747. ${ }^{1} \mathrm{H}$ NMR (400 MHz, DMSO-d $\left.\mathrm{d}_{6}\right): \delta 1.25\left(\mathrm{~s}, 3 \mathrm{H}, \mathrm{CH}_{3}\right), 1.53(\mathrm{~d}, 3 \mathrm{H}$, $\mathrm{CH}_{3}$ ), 3.88 (q, $\left.1 \mathrm{H}, \mathrm{CH}\right), 4.33$ (s, 2H, $\mathrm{NH}_{2}$ ), 7.00 (s, $1 \mathrm{H}, \mathrm{NH}$ ), 7.58-8.60 (m, 4H, Ar-H), $11.10(\mathrm{~s}, 1 \mathrm{H}, \mathrm{OH}) \mathrm{ppm} ;{ }^{13} \mathrm{C} \mathrm{NMR}$ $\left(100 \mathrm{MHz}, \mathrm{DMSO}-\mathrm{d}_{6}\right): \delta 26.16,27.30,42.68,72.35,99.49$, $121.21,123.74,136.02,146.08,158.55,174.67$ ppm. Anal. Calcd. for $\mathrm{C}_{11} \mathrm{H}_{15} \mathrm{~N}_{5} \mathrm{O}_{2}$ : C, 53.00, H, 6.07, N, 28.10. Found: C, 52.82, $\mathrm{H}, 6.09, \mathrm{~N}, 28.07$. 
3.2.2. 5b. 2-[3-Amino-5-methyl-5-(pyridin-3-yl)-1,5-dihydro4H-1,2,4-triazol-4-yl]butanedioic Acid. IR $\left(\mathrm{KBr}, \mathrm{cm}^{-1}\right) 3386$, 3263, 3034, 2634, 1610, 1504, 1270, 1089, 926, 704. ${ }^{1} \mathrm{H}$ NMR $\left(400 \mathrm{MHz}, \mathrm{DMSO}-\mathrm{d}_{6}\right): \delta 1.52\left(\mathrm{~s}, 3 \mathrm{H}, \mathrm{CH}_{3}\right), 3.03\left(\mathrm{~d}, 2 \mathrm{H}, \mathrm{CH}_{2}\right)$, $3.80(\mathrm{t}, 1 \mathrm{H}, \mathrm{CH}), 4.21\left(\mathrm{~s}, 2 \mathrm{H}, \mathrm{NH}_{2}\right), 7.15(\mathrm{~s}, 1 \mathrm{H}, \mathrm{NH}), 760-8.65$ (m, 4H, Ar-H), 10.82 (s, 2H, OH) ppm; ${ }^{13} \mathrm{C}$ NMR $(100 \mathrm{MHz}$, DMSO-d $\mathrm{d}_{6}$ ): $\delta 30.40,37.41,49.21,68.10,121.38,125.40,136.14$, $145.42,148.14,157.45,174.51,177.04 \mathrm{ppm}$. Anal. Calcd. for $\mathrm{C}_{12} \mathrm{H}_{15} \mathrm{~N}_{5} \mathrm{O}_{4}$ : C, 49.14, H, 5.16, N, 23.88. Found: C, 48.94, H, 5.18, N, 23.86.

3.2.3. 5c. 2-[3-Amino-5-methyl-5-(pyridin-3-yl)-1,5-dihydro4H-1,2,4-triazol-4-yl]-3-methyl Pentanoic Acid. IR (KBr, $\mathrm{cm}^{-1}$ ) 3371, 3264, 2967, 2619, 1608, 1586, 1394, 1187, 1089, 710. ${ }^{1} \mathrm{H}$ NMR $\left(400 \mathrm{MHz}\right.$, DMSO-d 6 ): $\delta 1.04\left(\mathrm{t}, 3 \mathrm{H}, \mathrm{CH}_{3}\right), 1.10$ $\left(\mathrm{d}, 3 \mathrm{H}, \mathrm{CH}_{3}\right), 1.68\left(\mathrm{~s}, 3 \mathrm{H}, \mathrm{CH}_{3}\right), 2.58(\mathrm{~m}, 1 \mathrm{H}, \mathrm{CH}), 3.03(\mathrm{~m}, 2 \mathrm{H}$, $\mathrm{CH}_{2}$ ), 3.88 (d, $\left.1 \mathrm{H}, \mathrm{CH}\right), 4.55$ (s, $\left.2 \mathrm{H}, \mathrm{NH}_{2}\right), 7.00(\mathrm{~s}, 1 \mathrm{H}, \mathrm{NH})$, 7.68-8.78 (m, 4H, Ar-H), 11.10 (s, 1H, OH) ppm; ${ }^{13} \mathrm{C}$ NMR $\left(100 \mathrm{MHz}, \mathrm{DMSO}-\mathrm{d}_{6}\right): \delta=13.42,16.32,24.01,28.75,32.45$, 49.47, 69.65, 120.30, 124.00, 136.42, 145.78, 148.36, 157.74, 177.50 ppm. Anal. Calcd. for $\mathrm{C}_{14} \mathrm{H}_{21} \mathrm{~N}_{5} \mathrm{O}_{2}: \mathrm{C}, 57.71, \mathrm{H}, 7.27$, N, 24.04. Found: C, 57.49, H, 7.26, N, 24.07.

3.2.4. 5d. 2-[3-Amino-5-methyl-5-(pyridin-3-yl)-1,5-dihydro4H-1,2,4-triazol-4-yl]-3-(1H-imidazol-4-yl)propanoic Acid. IR $\left(\mathrm{KBr}, \mathrm{cm}^{-1}\right)$ 3386, 3127, 2880, 2710, 1634, 1480, 1342, 1251, 1086, 923, 704. ${ }^{1} \mathrm{H}$ NMR $(400 \mathrm{MHz}$, DMSO-d 6 ): $\delta 1.83(\mathrm{~s}, 3 \mathrm{H}$, $\left.\mathrm{CH}_{3}\right), 3.42(\mathrm{t}, 1 \mathrm{H}, \mathrm{CH}), 2.58\left(\mathrm{~d}, 2 \mathrm{H}, \mathrm{CH}_{2}\right), 4.34\left(\mathrm{~s}, 2 \mathrm{H}, \mathrm{NH}_{2}\right)$, 7.40-7.93 (s, 2H, NH), 7.34-9.03 (m, 6H, Ar-H), 10.19 (s, H, OH) ppm; ${ }^{13} \mathrm{C}$ NMR $(100 \mathrm{MHz}$, DMSO-d 6 ): $\delta 29.58,30.45$, $52.10,70.46,110.24,121.38,122.45,125.47,136.14,144.42$, 147.21, 157.78, $177.45 \mathrm{ppm}$. Ms: $m / z$ : 315. Anal. Calcd. for $\mathrm{C}_{14} \mathrm{H}_{17} \mathrm{~N}_{7} \mathrm{O}_{2}$ : C, 53.32, H, 5.43, N, 31.09. Found: C, 53.93, H, $5.45, \mathrm{~N}, 31.74$.

3.2.5. 5e. 2-[3-Amino-5-methyl-5-(pyridin-3-yl)-1,5-dihydro4H-1,2,4-triazol-4-yl]-4-(methyl sulfanyl)butanoic Acid. IR $\left(\mathrm{KBr}, \mathrm{cm}^{-1}\right) 3386,3263,2917,2610,1611,1506,1494,1089,975$, 704. ${ }^{1} \mathrm{H}$ NMR $\left(400 \mathrm{MHz}\right.$, DMSO-d 6 ): $\delta 1.94\left(\mathrm{~s}, 3 \mathrm{H}, \mathrm{CH}_{3}\right)$, $1.96\left(\mathrm{~s}, 3 \mathrm{H}, \mathrm{CH}_{3}\right), 2.06\left(\mathrm{~m}, 2 \mathrm{H}, \mathrm{CH}_{2}\right), 2.55\left(\mathrm{~d}, 2 \mathrm{H}, \mathrm{CH}_{2}\right), 3.48$ (t, $1 \mathrm{H}, \mathrm{CH}), 4.65\left(\mathrm{~s}, 2 \mathrm{H}, \mathrm{NH}_{2}\right), 7.10(\mathrm{~s}, 1 \mathrm{H}, \mathrm{NH}), 7.66-8.67$ (m, 4H, Ar-H), 10.22 (s, $1 \mathrm{H}, \mathrm{OH}) \mathrm{ppm} ;{ }^{13} \mathrm{C}$ NMR $(100 \mathrm{MHz}$, DMSO-d $\mathrm{d}_{6}$ ): $\delta 14.81,26.06,29.03,30.45,49.47,66.78,124.47$, 136.56, 147.30, 145.45, 156.41, $177.54 \mathrm{ppm}$. Anal. Calcd. for $\mathrm{C}_{13} \mathrm{H}_{19} \mathrm{~N}_{5} \mathrm{O}_{2} \mathrm{~S}$ : C, 50.47, H, 6.19, N, 22.64. Found: C, 50.28, $\mathrm{H}, 6.18, \mathrm{~N}, 22.67$.

3.2.6. 5f. 2-[3-Amino-5-methyl-5-(pyridin-3-yl)-1,5-dihydro4H-1,2,4-triazol-4-yl]-3-phenyl Propanoic Acid. IR (KBr, $\mathrm{cm}^{-1}$ ) 3402, 3202, 2545, 1611, 1455, 1315, 1098, 856, 739. ${ }^{1} \mathrm{H}$ NMR $\left(400 \mathrm{MHz}, \mathrm{DMSO}-\mathrm{d}_{6}\right): \delta 1.24\left(\mathrm{~s}, 3 \mathrm{H}, \mathrm{CH}_{3}\right), 2.36(\mathrm{~d}$, $\left.2 \mathrm{H}, \mathrm{CH}_{2}\right), 2.58(\mathrm{t}, 1 \mathrm{H}, \mathrm{CH}), 4.92\left(\mathrm{~s}, 2 \mathrm{H}, \mathrm{NH}_{2}\right), 7.23(\mathrm{~s}, 1 \mathrm{H}$, $\mathrm{NH}), 7.48-9.19(\mathrm{~m}, 9 \mathrm{H}, \mathrm{Ar}-\mathrm{H}), 10.28(\mathrm{~s}, 1 \mathrm{H}, \mathrm{OH}) \mathrm{ppm} ;{ }^{13} \mathrm{C}$ NMR $\left(100 \mathrm{MHz}\right.$, DMSO-d $\left.{ }_{6}\right): \delta 30.04,35.37,51.52,65.12$, $123.04,125.45,127.54,128.65,135.41,139.08,147.25,140.36$, $149.32,154.23,177.90$ ppm. Anal. Calcd. for $\mathrm{C}_{17} \mathrm{H}_{19} \mathrm{~N}_{5} \mathrm{O}_{2}$ : C, 62.75, H, 5.89, N, 21.52. Found: C, 62.55, H, 5.91, N, 21.48.
3.2.7. 5g. 2-[3-Amino-5-methyl-5-(pyridin-3-yl)-1,5-dihydro4H-1,2,4-triazol-4-yl]-3-hydroxy Propanoic Acid. IR (KBr, $\mathrm{cm}^{-1}$ ) 3421, 3320, 3264, 2879, 2603, 1647, 1556, 1354, 1207, 1075, 710. ${ }^{1} \mathrm{H}$ NMR $\left(400 \mathrm{MHz}\right.$, DMSO- $\left.\mathrm{d}_{6}\right): \delta 1.50\left(\mathrm{~s}, 3 \mathrm{H}, \mathrm{CH}_{3}\right), 3.58$ $(\mathrm{t}, 1 \mathrm{H}, \mathrm{CH}), 3.90\left(\mathrm{~d}, 2 \mathrm{H}, \mathrm{CH}_{2}\right), 4.71\left(\mathrm{~s}, 2 \mathrm{H}, \mathrm{NH}_{2}\right), 7.08(\mathrm{~s}, 1 \mathrm{H}$, $\mathrm{NH}), 7.42-8.75(\mathrm{~m}, 4 \mathrm{H}, \mathrm{Ar}-\mathrm{H}), 9.50(\mathrm{~s}, 1 \mathrm{H}, \mathrm{OH}), 11.05(\mathrm{~s}, 1 \mathrm{H}$, OH) ppm; ${ }^{13} \mathrm{C}$ NMR $\left(100 \mathrm{MHz}, \mathrm{DMSO}-\mathrm{d}_{6}\right): \delta 30.21,52.45$, 65.02, 123.09, 135.14, 139.20, 147.34, 149.40, 154.03, $176.21 \mathrm{ppm}$. Anal. Calcd. for $\mathrm{C}_{11} \mathrm{H}_{15} \mathrm{~N}_{5} \mathrm{O}_{3}$ : C, 49.81, H, 5.70, N, 26.40 . Found: C, 49.58, H, 5.68, N, 26.42.

3.2.8. 5h. 2-[3-Amino-5-methyl-5-(pyridin-3-yl)-1,5-dihydro4H-1,2,4-triazol-4-yl]-3-(1H-indol-3-yl)propanoic Acid. IR $\left(\mathrm{KBr}, \mathrm{cm}^{-1}\right) 3402,3202,2545,1611,1481,1315,1056,739 .{ }^{1} \mathrm{H}$ NMR $\left(400 \mathrm{MHz}, \mathrm{DMSO}-\mathrm{d}_{6}\right): \delta 1.10\left(\mathrm{~s}, 3 \mathrm{H}, \mathrm{CH}_{3}\right), 2.90(\mathrm{~d}$, $\left.2 \mathrm{H}, \mathrm{CH}_{2}\right), 3.82(\mathrm{t}, 1 \mathrm{H}, \mathrm{CH}), 4.82\left(\mathrm{~s}, 2 \mathrm{H}, \mathrm{NH}_{2}\right), 7.10(\mathrm{~s}, 1 \mathrm{H}$, $\mathrm{NH}), 7.20-8.65$ (m, 9H, Ar-H), $9.85(\mathrm{~s}, 1 \mathrm{H}, \mathrm{NH}), 10.48(\mathrm{~s}, 1 \mathrm{H}$, $\mathrm{OH}) \mathrm{ppm} ;{ }^{13} \mathrm{C}$ NMR $\left(100 \mathrm{MHz}, \mathrm{DMSO}-\mathrm{d}_{6}\right): \delta 28.41,30.64$, 56.21, 70.12, 110.04, 111.12, 118.14, 120.10, 121.16, 122.45, 124.40, $127.23,136.20,147.45,154.24,158.60,174.57$ ppm. Anal. Calcd. for $\mathrm{C}_{19} \mathrm{H}_{20} \mathrm{~N}_{6} \mathrm{O}_{2}$ : C, 62.62, H, 5.53, N, 23.06. Found: C, 62.40, $\mathrm{H}$, 5.51, N, 23.09.

3.2.9. 5i. 2-[3-Amino-5-methyl-5-(pyridin-3-yl)-1,5-dihydro4H-1,2,4-triazol-4-yl]-3-methyl Butanoic Acid. IR (KBr, $\mathrm{cm}^{-1}$ ) 3356, 3247, 2817, 2634, 1611, 1583, 1374, 1177, 1059, 983, 708. ${ }^{1} \mathrm{H}$ NMR $(400 \mathrm{MHz}$, DMSO-d 6 ): $\delta 1.01(\mathrm{~d}, 6 \mathrm{H}, 2 \times$ $\left.\mathrm{CH}_{3}\right), 1.52\left(\mathrm{~s}, 3 \mathrm{H}, \mathrm{CH}_{3}\right), 2.39(\mathrm{~m}, 1 \mathrm{H}, \mathrm{CH}), 3.48(\mathrm{~d}, 1 \mathrm{H}, \mathrm{CH})$, $4.65\left(\mathrm{~s}, 2 \mathrm{H}, \mathrm{NH}_{2}\right), 7.00(\mathrm{~s}, 1 \mathrm{H}, \mathrm{NH}), 7.42-7.88(\mathrm{~m}, 4 \mathrm{H}, \mathrm{Ar}-\mathrm{H})$, $11.02(\mathrm{~s}, 1 \mathrm{H}, \mathrm{OH}) \mathrm{ppm} ;{ }^{13} \mathrm{C}$ NMR $\left(100 \mathrm{MHz}\right.$, DMSO$\left.-\mathrm{d}_{6}\right): \delta$ 16.91, 25.23, 30.24, 65.47, 123.10, 135.42, 139.10, 147.32, 154.06, 177.20 ppm. Anal. Calcd. for $\mathrm{C}_{13} \mathrm{H}_{19} \mathrm{~N}_{5} \mathrm{O}_{2}$ : C, 56.30, H, 6.91, N, 25.25. Found: C, 56.22, H, 6.92, N, 25.22.

3.2.10. 9a. Ethyl-2-[3-amino-5-methyl-5-(pyridin-3-yl)-1,5dihydro-4H-1,2,4-triazol-4-yl]-3-sulfanylpropanoate. IR ( $\mathrm{KBr}$, $\mathrm{cm}^{-1}$ ) 3311, 3217, 2937, 1618, 1556, 1373, 1198, 1089, 735. ${ }^{1} \mathrm{H}$ NMR $\left(400 \mathrm{MHz}, \mathrm{DMSO}-\mathrm{d}_{6}\right): \delta 1.30\left(\mathrm{t}, 3 \mathrm{H}, \mathrm{CH}_{3}\right), 1.24(\mathrm{~s}$, $1 \mathrm{H}, \mathrm{S}-\mathrm{H}), 1.52\left(\mathrm{~s}, 3 \mathrm{H}, \mathrm{CH}_{3}\right), 3.03\left(\mathrm{~s}, 2 \mathrm{H}, \mathrm{CH}_{2}\right), 3.77$ (s, $1 \mathrm{H}, \mathrm{CH}), 4.14\left(\mathrm{q}, 2 \mathrm{H}, \mathrm{CH}_{2}\right), 4.85\left(\mathrm{~s}, 2 \mathrm{H}, \mathrm{NH}_{2}\right), 7.08(\mathrm{~s}, 1 \mathrm{H}$, $\mathrm{NH}), 7.42-7.88(\mathrm{~m}, 4 \mathrm{H}, \mathrm{Ar}-\mathrm{H}) \mathrm{ppm} ;{ }^{13} \mathrm{C}$ NMR $(100 \mathrm{MHz}$, DMSO-d $\left.{ }_{6}\right): \delta 13.64,25.32,30.45,51.45,59.42,64.23,123.14$, 135.40, 139.30, 147.45, 149.10, 154.20, 173.15 ppm. Anal. Calcd. for $\mathrm{C}_{13} \mathrm{H}_{19} \mathrm{~N}_{5} \mathrm{O}_{2} \mathrm{~S}: \mathrm{C}, 50.47, \mathrm{H}, 6.19, \mathrm{~N}, 22.64$. Found: C, 50.27, $\mathrm{H}, 6.23, \mathrm{~N}, 22.67$.

\section{Conclusion}

The use of water as a green solvent and alum as a green catalyst offers a convenient, nontoxic, inexpensive approach for the synthesis of triazole derivatives. This procedure is simpler, economical, milder, and faster, including cleaner reactions, high yields of products, and a simple experimental and workup procedure, which makes it a useful and attractive process and is also consistent with the green chemistry theme which affords good yields. 


\section{Conflict of Interests}

The authors declare that there is no conflict of interests regarding the publication of this paper.

\section{Acknowledgments}

The authors are thankful to the Dean, FET, MITS, for providing necessary research facilities in the department. Financial assistance from FET, MITS, is gratefully acknowledged. They are also thankful to the Director, SAIF, Punjab University, Chandigarh, for the spectral and elemental analyses.

\section{References}

[1] S. S. Pawar, M. S. Shingare, and S. N. Thore, "A novel approach for ligand promoted palladium (II)-catalyzed suzuki coupling of aryl iodides and bromides with arylboronic acid in aqueous media," Letters in Organic Chemistry, vol. 4, no. 7, pp. 486-490, 2007.

[2] K. Gong, Z.-W. He, Y. Xu, D. Fang, and Z.-L. Liu, "Green synthesis of 5-benzylidene rhodanine derivatives catalyzed by 1-butyl3-methyl imidazolium hydroxide in water," Monatshefte für Chemie, vol. 139, no. 8, pp. 913-915, 2008.

[3] A. Pinaka, G. C. Vougioukalakis, D. Dimotikali, E. Yannakopoulou, B. Chankvetadze, and K. Papadopoulos, "Green asymmetric synthesis: b-amino alcohol-catalyzed direct asymmetric aldol reactions in aqueous micelles," Chirality, vol. 25, no. 2, pp. 119-125, 2013.

[4] A. A. Mohammadi, A. Hadadzahmatkesh, and M. R. Asghariganjeh, " $\mathrm{FeNH}_{4}\left(\mathrm{SO}_{4}\right)_{2} \cdot 12 \mathrm{H}_{2} \mathrm{O}$ (alum)-catalyzed preparation of 1,4-dihydropyridines: improved conditions for the Hantzsch reaction," Monatshefte für Chemie, vol. 143, no. 6, pp. 931-933, 2012.

[5] J. Azizian, A. A. Mohammadi, A. E. Karimi, and M. R. Mohammadizadeh, "A stereoselective three-component reaction: $\left(\mathrm{KAl}\left(\mathrm{SO}_{4}\right)_{2} \cdot 12 \mathrm{H}_{2} \mathrm{O}\right)$, an efficient and reusable catalyst for the one-pot synthesis of cis-isoquinolonic acids," Journal of Organic Chemistry, vol. 70, no. 1, pp. 350-352, 2005.

[6] M. Dabiri, P. Salehi, S. Otokesh, M. Baghbanzadeh, G. Kozehgary, and A. A. Mohammadi, "Efficient synthesis of monoand disubstituted 2,3-dihydroquinazolin-4(1H)-ones using $\left(\mathrm{KAl}\left(\mathrm{SO}_{4}\right)_{2} \cdot 12 \mathrm{H}_{2} \mathrm{O}\right)$ as a reusable catalyst in water and ethanol," Tetrahedron Letters, vol. 46, no. 36, pp. 6123-6126, 2005.

[7] J. Azizian, A. A. Mohammadi, A. R. Karimi, and M. R. Mohammadizadeh, " $\left(\mathrm{KAl}\left(\mathrm{SO}_{4}\right)_{2} \cdot 12 \mathrm{H}_{2} \mathrm{O}\right)$ supported on silica gel as a novel heterogeneous system catalyzed biginelli reaction: onepot synthesis of di-hydropyrimidinones under solvent-free conditions," Applied Catalysis A, vol. 300, no. 1, pp. 85-88, 2006.

[8] J. Azizian, A. A. Mohammadi, I. Bidar, and P. Mirzaei, " $\left(\mathrm{KAl}\left(\mathrm{SO}_{4}\right)_{2} \cdot 12 \mathrm{H}_{2} \mathrm{O}\right)$ (alum) a reusable catalyst for the synthesis of some 4-substituted coumarins via Pechmann reaction under solvent-free conditions," Monatshefte für Chemie, vol. 139, no. 7, pp. 805-808, 2008.

[9] K. F. Shelke, S. B. Sapkal, G. K. Kakade, S. A. Sadaphal, B. B. Shingate, and M. S. Shingare, "Alum catalyzed simple and efficient synthesis of 5-arylidene-2,4-thiazolidinedione in aqueous media," Green Chemistry Letters and Reviews, vol. 3, no. 1, pp. 17-21, 2010.

[10] M. Dabiri, M. Baghbanzadeh, M. S. Nikcheh, and E. Arzroomchilar, "Eco-friendly and efficient one-pot synthesis of alkyl- or aryl-14H-dibenzo[a,j]xanthenes in water," Bioorganic and Medicinal Chemistry Letters, vol. 18, no. 1, pp. 436-438, 2008.

[11] D. Mahajan, T. Naqvi, R. L. Sharma, and K. K. Kapoor, "Alumcatalyzed one-pot solventless synthesis of 1,5-benzodiazepines," Australian Journal of Chemistry, vol. 61, no. 2, pp. 159-162, 2008.

[12] A. A. Mohammadi, M. Mivechi, and H. Kefayati, "Potassium aluminum sulfate (alum): an efficient catalyst for the one-pot synthesis of trisubstituted imidazoles," Monatshefte für Chemie, vol. 139, no. 8, pp. 935-937, 2008.

[13] H. Bayrak, A. Demirbas, S. A. Karaoglu, and N. Demirbas, "Synthesis of some new 1,2,4-triazoles, their Mannich and Schiff bases and evaluation of their antimicrobial activities," European Journal of Medicinal Chemistry, vol. 44, no. 3, pp. 1057-1066, 2009.

[14] B. S. Holla, M. Mahalinga, M. S. Karthikeyan, B. Poojary, P. M. Akberali, and N. S. Kumari, "Synthesis, characterization and antimicrobial activity of some substituted 1,2,3-triazoles," European Journal of Medicinal Chemistry, vol. 40, no. 11, pp. 11731178, 2005.

[15] S. Bhimagouda Patil, G. Krishnamurthy, N. D. Shashikumar, M. R. Lokesh, and H. S. Bhojya Naik, "Synthesis and antimicrobial activity of some [1,2,4]-triazole derivatives," Journal of Chemistry, vol. 2013, Article ID 462594, 7 pages, 2013.

[16] U. Salgin-Gökşen, N. Gökhan-Kelekçi, Ö. Göktaş et al., "1Acylthiosemicarbazides, 1,2,4-triazole-5(4H)-thiones, 1,3,4-thiadiazoles and hydrazones containing 5-methyl-2-benzoxazolinones: synthesis, analgesic-anti-inflammatory and antimicrobial activities," Bioorganic \& Medicinal Chemistry, vol. 15, no. 17, pp. 5738-5751, 2007.

[17] M. S. Karthikeyan, "Synthesis, analgesic, anti-inflammatory and antimicrobial studies of 2,4-dichloro-5-fluorophenyl containing thiazolotriazoles," European Journal of Medicinal Chemistry, vol. 44, no. 2, pp. 827-833, 2009.

[18] K. Sztanke, T. Tuzimski, J. Rzymowska, K. Pasternak, and M. Kandefer-Szerszeń, "Synthesis, determination of the lipophilicity, anticancer and antimicrobial properties of some fused 1,2,4triazole derivatives," European Journal of Medicinal Chemistry, vol. 43, no. 2, pp. 404-419, 2008.

[19] K. Ilango and P. Valentina, "Synthesis and biological activities of novel 1,2,4-triazolo-[3,4-b]-1,3,4-thiadiazoles," Der Pharma Chemica, vol. 2, no. 2, pp. 16-22, 2010.

[20] S. Crotty, D. Maag, J. J. Arnold et al., "The broad-spectrum antiviral ribonucleoside ribavirin is an RNA virus mutagen," Nature Medicine, vol. 6, no. 12, pp. 1375-1379, 2000.

[21] D. J. Williamson, R. G. Hill, S. L. Shepheard, and R. J. Hargreaves, "The anti-migraine 5-HT1B/1D agonist rizatriptan inhibits neurogenic dural vasodilation in anaesthetized guineapigs," British Journal of Pharmacology, vol. 133, no. 7, pp. 10291034, 2001.

[22] Y. K. Lee and A. W. Fothergill, "In vitro antifungal activities of amphotericin B, fluconazole, itraconazole, terbinafine, caspofungin, voriconazole, and posaconazole against 30 clinical isolates of cryptococcus neoformans var. neoformancs," Mycobiology, vol. 31, no. 2, pp. 95-98, 2003.

[23] H. A. Torres, R. Y. Hachem, R. F. Chemaly, D. P. Kontoyiannis, and I. I. Raad, "Posaconazole: a broad-spectrum triazole antifungal," The Lancet Infectious Diseases, vol. 5, no. 12, pp. 775785, 2005.

[24] M. A. Al-Omar, E. S. Al-Abdullah, I. A. Shehata, E. E. Habib, T. M. Ibrahim, and A. A. El-Emam, "Synthesis, antimicrobial, 
and anti-inflammatory activities of novel 5-(1-adamantyl)-4arylideneamino-3-mercapto-1,2,4-triazoles and related derivatives," Molecules, vol. 15, no. 4, pp. 2526-2550, 2010.

[25] N. Demirbaş, R. Ugurluoglu, and A. Demirbaş, "Synthesis of 3-alkyl(Aryl)-4-alkylidenamino-4,5-dihydro-1H-1,2,4-triazol5-ones and 3-alkyl-4-alkylamino-4,5-dihydro-1H-1,2,4-triazol5-ones as antitumor agents," Bioorganic \& Medicinal Chemistry, vol. 10, no. 12, pp. 3717-3723, 2002.

[26] B. Kahveci, O. Bekircan, M. Serdar, and A. A. Ikizler, "Synthesis of some 4-(arylmethylamino)-4,5-dihydro-1H-1,2,4-triazol-5ones," Indian Journal of Chemistry Section B, vol. 42, no. 6, pp. 1527-1530, 2003.

[27] N. Demirbaş and R. Uğurluoğlu, "Synthesis of novel 4alkylidene- and 4-alkylamino-5-oxo-4,5-dihydro-[1,2,4] triazole derivatives and investigation of their antitumor activities," Turkish Journal of Chemistry, vol. 28, no. 5, pp. 559-571, 2004.

[28] O. Bekircan, B. Kahveci, and M. Küçük, "Synthesis and anticancer evaluation of some new unsymmetrical 3,5-diaryl-4H1,2,4-triazole derivatives," Turkish Journal of Chemistry, vol. 30, no. 1, pp. 29-40, 2006.

[29] H. Yuksek, S. Kolayli, M. Kucuk et al., "Synthesis and antioxidant activities of some 4-benzyl-idenamino-4, 5-dihydro-1H1, 2, 4-triazol-5-one derivatives," Indian Journal of Chemistry. Section B, vol. 45, no. 3, p. 715, 2006.

[30] A. Dandia, R. Singh, H. Sachdeva, and K. Arya, "Microwave assisted one pot synthesis of a series of trifluoromethyl substituted spiro [indole-triazoles]," Journal of Fluorine Chemistry, vol. 111, no. 1, pp. 61-67, 2001.

[31] A. Dandia, H. Sachdeva, and R. Singh, "Improved synthesis of 3-spiro indolines in dry media under microwave irradiation," Synthetic Communications, vol. 31, no. 12, pp. 1879-1892, 2001.

[32] H. Sachdeva and D. Dwivedi, "Lithium-acetate-mediated biginelli one-pot multicomponent synthesis under solvent-free conditions and cytotoxic activity against the human lung cancer cell line A549 and breast cancer cell line MCF7,' The Scientific World Journal, vol. 2012, Article ID 109432, 9 pages, 2012.

[33] H. Sachdeva, R. Saroj, S. Khaturia, and D. Dwivedi, "Environeconomic synthesis and characterization of some new 1, 2, 4triazole derivatives as organic fluorescent materials and potent fungicidal agents," Organic Chemistry International, vol. 2013, Article ID 659107, 19 pages, 2013. 

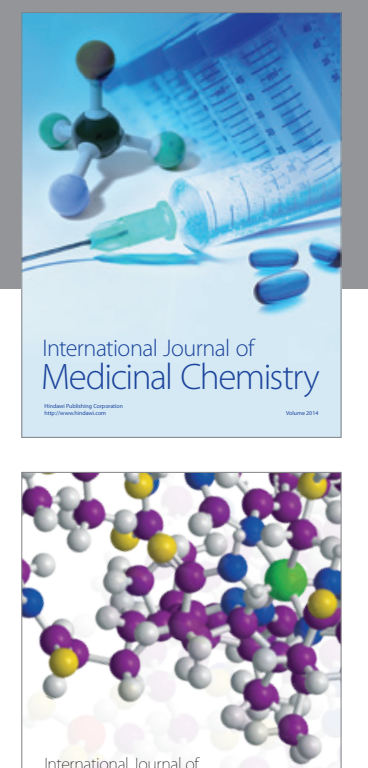

\section{Carbohydrate} Chemistry

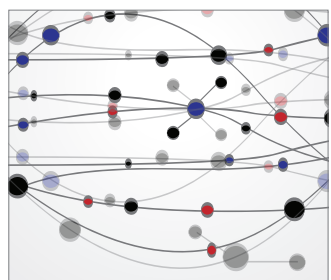

The Scientific World Journal
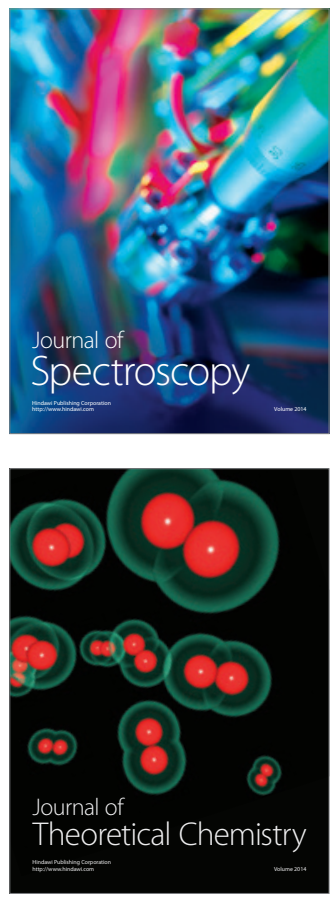
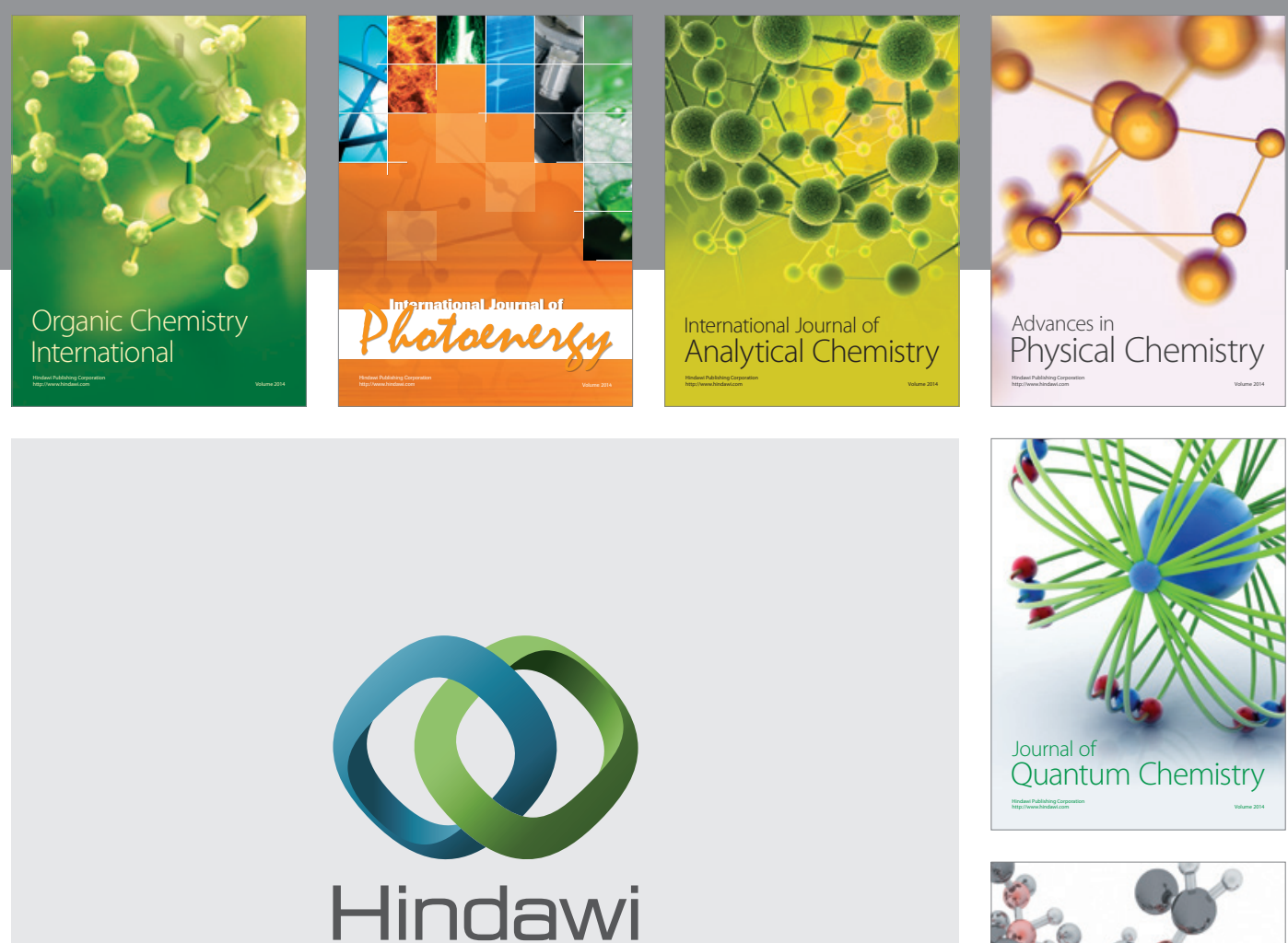

Submit your manuscripts at

http://www.hindawi.com

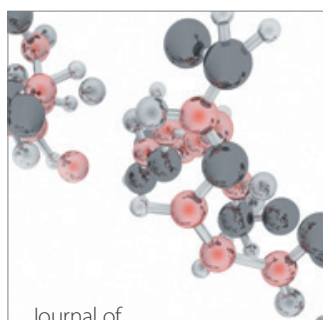

Analytical Methods

in Chemistry

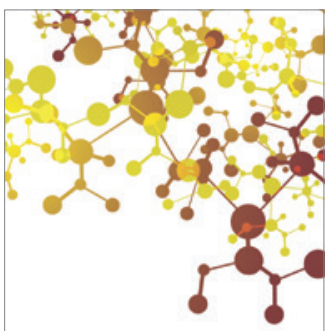

Journal of

Applied Chemistry

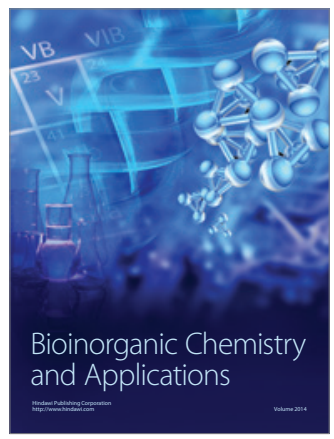

Inorganic Chemistry
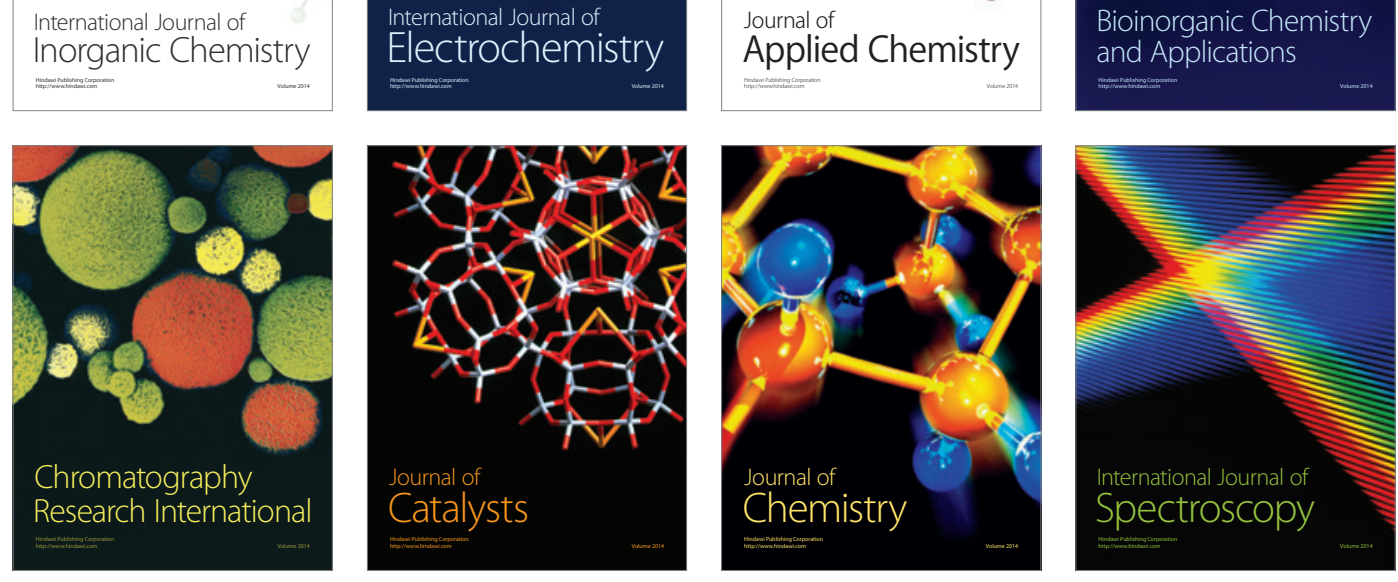